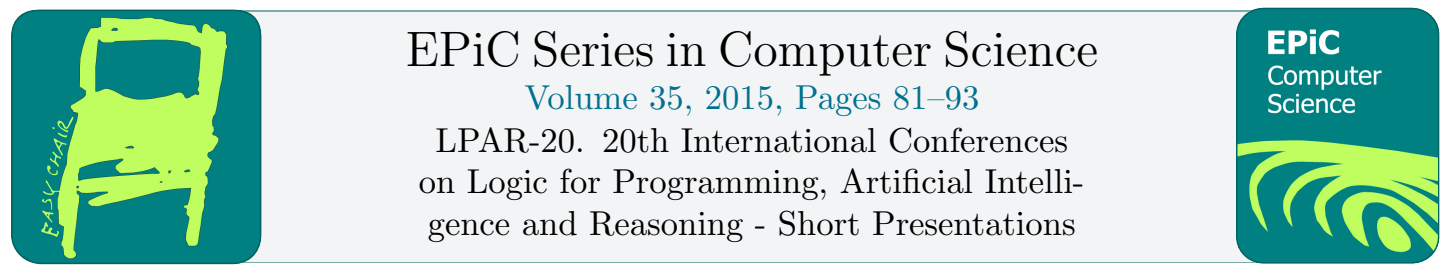

\title{
A Lightweight Double-negation Translation
}

\author{
Frédéric Gilbert \\ École des Ponts ParisTech, Inria, CEA LIST \\ frederic.a.gilbert@inria.fr
}

\begin{abstract}
Deciding whether a classical theorem can be proved constructively is a well-known undecidable problem. As a consequence, any computable double-negation translation inserts some unnecessary double negations. This paper shows that most of these unnecessary insertions can be avoided without any use of constructive proof search techniques. For this purpose, we restrict the analysis to syntaxdirected double-negation translations, which translate a proposition through a single traversal - and include most of the usual translations such as Kolmogorov's, Gödel-Gentzen's, and Kuroda's. A partial order among translations are presented to select translations avoiding as many double negations as possible. This order admits a unique minimal syntax-directed translation with noticeable properties.
\end{abstract}

\section{Introduction}

The status of the law of excluded middle in a proof system has significant implications: classical systems admit faster proof search algorithms, while constructive systems have more straightforward proofs-as-programs interpretations. As more and more systems implementing classical and constructive logic are developed, the question of translating theorems or proofs from one to the other becomes relevant. Translating constructive logic into classical logic is easy, as constructive theorems and proofs are a fortiori classical ones, but all distinctive properties of constructive proofs, such as the witness property, are lost in such translations. Thus, it seems more interesting to go in the other direction. However, as not all classical theorems are provable constructively - this question is even undecidable - classical logic can be embedded only through a correct translation, i.e. a function mapping classical theorems to constructive ones. In first-order logic, a usual way to translate a classical theorem is to insert double negations in it. Based on this idea, many correct double-negation translations have been developed; Kolmogorov's [6], Gödel-Gentzen's [4, 5] and Kuroda's [8] can be mentioned among them - the associated proofs translations are thoroughly studied in [10].

In order to push interoperability between classical and constructive systems as far as possible, we want to transpose from classical to constructive logic not only absolute provability but also provability relative to a given context. For this purpose, a translation $f$ will be given by a pair of functions $\left(f^{+}, f^{-}\right)$, with $f^{+}$mapping propositions to propositions and $f^{-}$mapping contexts to contexts, defining $f(\Gamma \vdash C)$ as $f^{-}(\Gamma) \vdash f^{+}(C)$. This definition based on sequents instead of propositions is more expressive at the cost of some extra restrictions. Using a definition restricted to propositions, i.e. to absolute provability, all unprovable propositions are 
considered the same: for instance, any atomic proposition can be mapped to $\perp$. Using this definition based on sequents, such translations are not allowed anymore. Many double-negation translations presented as translations of propositions can fit the sequents-based definition, using the same function to translate propositions and contexts - Kolmogorov's, Gödel-Gentzen's, and Kuroda's can be mentioned among them. Other translations as in [1] are designed to transpose absolute provability only and cannot be transposed to correct sequent-based translations in such a way.

The intention in this paper is to present sequent-based translations that are not only correct but also as faithful as possible: indeed, the most convenient way to reuse a classical proof in a constructive context is to translate it to a constructive judgment chosen as compliant as possible to the original one. For instance, a translation mapping all provable propositions to $\top$ wouldn't be very useful when trying to reuse classical theorems or proofs in a constructive context. As double-negation translations alter sequents in a more limited way, they are better suited to fit these restrictions. For this reason, we choose to focus in this paper on double-negations translations only.

Given a sequent $S$, two characteristics of $S$ will be used as a bases for compliance comparisons: its syntax, and the set of judgments that derive from it constructively. Given a double-negation translation $S^{\prime}$ of $S$, the first standpoint suggests that $S^{\prime}$ should contain as few inserted double negations as possible, while the second suggests that as many sequents as possible should be either derivable from both $S$ and $S^{\prime}$ or from none of them. In the following, the effect of a double negation translation from the first standpoint will be denoted as the syntax alteration, while its effect from the second standpoint will be denoted as the strength alteration. For instance, among all translations of $\vdash \neg \neg P \Rightarrow P$, the syntax alteration is better in $\vdash \neg \neg P \Rightarrow \neg \neg P$ than in $\vdash \neg \neg(\neg \neg P \Rightarrow \neg \neg P)$, but, as both translations are constructively equivalent, the strength alteration is the same.

These compliance requirements are not sufficient to ensure a genuine interoperability between classical and constructive logic. In particular, we don't consider as a tool of interoperability an algorithm from classical proofs to constructive ones that discard its argument and builds its image from scratch using constructive proof search algorithms - even if this kind of translation would be potentially good with respect to compliance requirements. For this reason, we will select translations avoiding any use of constructive proof search techniques.

The purpose of this paper is to determine to what extent the images of double-negation translations can be faithful to the original ones without using constructive theorem proving techniques. First, a set of double-negation translations fitting this restriction is presented. It is denoted as the set of syntax-directed translations, and contains several usual translations such as Kolmogorov's, Gödel-Gentzen's, and Kuroda's. Then, we will show that translations avoiding as many double-negations as possible can be considered to alter less the strength of sequents as well as their syntax. This observation allows to define two partial orders of double negation translations, the second being an extension of the first. The extended partial order leads to a unique minimal translation among correct syntax-directed translations, which avoids many unnecessary double negation insertions compared to the usual ones.

Some of the proofs are limited to key ideas due to space constraints; the complete proofs will be available in the extended version of the paper.

\section{Syntax-directed double-negation translations}

This paper is about classical and constructive first-order predicate logic, presented in the sequent calculi $L K$ and $L J$. The connectives and quantifier are the usual: $\wedge, \vee, \Rightarrow, \neg, \forall, \exists, \perp, \top$. In 
the current context, it is important to refrain from applying classical equivalences to eliminate one of them, for instance defining $A \Rightarrow B$ as $B \vee \neg A$ : keeping the distinction between both is crucial to be able to translate them in different ways in constructive logic.

The purpose of this section is to discard double-negation translations that might be based on constructive theorem proving techniques: as mentioned earlier, the benefit of translating classical proofs to constructive ones would be lost if constructive proofs were built from constructive theorem proving techniques, without using the data of the original classical proofs. We want to exclude translations such as, for instance, uncomputable ones mapping any constructively provable sequent to itself.

In sequent calculus without cuts, the subformula property holds: the proof of a sequent is built from proofs of sequents involving subformulae of the original sequent exclusively. On one hand, the leafs of the proof are built from the identification of disjoint subformulae. On the other hand, the inner nodes are built recursively, from deep to shallow subformulae. Therefore, an effective way to avoid any constructive theorem proving computation is to decide the number of double negation inserted in front of a given subformula regardless of the content of any disjoint subformulae on one hand, and regardless of any strictly included subformulae on the other hand. Furthermore, following the same idea, the translation of a proposition in a context $\Gamma$ must be done regardless of the presence of other propositions in $\Gamma: f^{-}\left(C_{1}, \ldots, C_{n}\right)=f^{-}\left(C_{1}\right), \ldots, f^{-}\left(C_{n}\right)$.

In a given sequent, the remaining information available to decide the number of double negations inserted at a given occurence is contained in the path going from the root of the sequent to this occurence. Therefore, such translations can be computed in a single traversal of the sequent beginning at its root - a necessary and sufficient condition is that any node is discovered before its children. For this reason, these translations will be denoted as syntaxdirected translations.

The path going from the root of a sequent to one of its occurences contains the following information: the place of the proposition containing the occurence in the sequent (antecedent or consequent), the labels of the nodes going from the root of this proposition to the occurence (connectives, quantifiers, or predicate symbols), and, for every binary connective crossed above the occurence, which direct child contains the occurence (left one or right one). We formalize this notion of path in the following way, using the usual notations of regular expressions:

Definition 1. - The place of a proposition in a sequent is noted (-) for antecedents and (+) for consequents.

- The set of predicate symbols is noted $S$. We define the set of labels $L$ as the following: $(\wedge|\vee| \Rightarrow|\neg| \forall|\exists| \perp|\top| S)$.

- The indication of a direct child of a proposition beginning with a binary connective is noted $\mathrm{L}$ for the left one and $\mathrm{R}$ for the right one. We define the language $\mathrm{E}$ of directed edges: $(\wedge \mathrm{L}|\wedge \mathrm{R}| \vee \mathrm{L}|\vee \mathrm{R}| \Rightarrow \mathrm{L}|\Rightarrow \mathrm{R}| \neg|\forall| \exists)$.

- The language of $P$ paths is the following: $(-\mid+) E^{*} L$

For instance, the path corresponding to an atomic proposition $P$ in the sequent $A \wedge(\neg P \vee$ $C) \vdash D$ is $(-\wedge \mathrm{R} \vee \mathrm{L} \neg P)$.

A syntax-directed translation inserts double-negations at a given occurence according to the path leading to it. Therefore, it can be given by a multiset of paths: for each path, the number of double negations inserted at the occurence corresponding to this path is its multiplicity in the multiset. Formally, the link between a multiset of paths and its corresponding syntax-directed translation is the following: 
Definition 2. Let $X$ be a multiset of paths. Let $w \in(-\mid+) E^{*}$. Let $A$ be a proposition. We define the proposition $F_{X}(w, A)$ recursively as follows.

Let $l_{A}$ be the label at the root of $A$. Let $n(w, A, X)$ be the multiplicity of $w l_{A}$ in $X$.

- If $l_{A} \in(\perp|\top| S), F_{X}(w, A)=(\neg \neg)^{n(w, A, X)} A$.

- If $l_{A} \in(\neg|\forall| \exists), F_{X}(w, A)=(\neg \neg)^{n(w, A, X)} l_{A}\left(F_{X}\left(w l_{A}, A_{\mathrm{C}}\right)\right)$, where $A=l_{A}\left(A_{\mathrm{C}}\right)$.

- If $l_{A} \in(\wedge|\vee| \Rightarrow), F_{X}(w, A)=(\neg \neg)^{n(w, A, X)} l_{A}\left(F_{X}\left(w l_{A} \mathrm{~L}, A_{\mathrm{L}}\right), F_{X}\left(w l_{A} \mathrm{R}, A_{\mathrm{R}}\right)\right)$, where $A=l_{A}\left(A_{\mathrm{L}}, A_{\mathrm{R}}\right)$.

For any multiset of paths $X$, we define $f_{X}^{-}, f_{X}^{+}$, and $f_{X}$ as follows:

- $f_{X}^{+}(A)=F_{X}(+, A)$ and $f_{X}^{-}(A)=F_{X}(-, A)$

- $f_{X}^{-}\left(A_{1}, \ldots, A_{n}\right)=f_{X}^{-}\left(A_{1}\right), \ldots, f_{X}^{-}\left(A_{n}\right)$

- $f(\Gamma \vdash C)=f^{-}(\Gamma) \vdash f^{+}(C)$

$f_{X}$ is is referred as the syntax-directed translation associated with $X$.

Example 1. Kolmogorov's, Gödel-Gentzen's, and Kuroda's double negation translations are all syntax-directed translations.

- Kolmogorov double-negation translation consists in the insertion of double negations at all occurences: the corresponding multiset is the set of all paths $X_{K o}=P$.

- Gödel-Gentzen double-negation translation consists in the insertion of double negations in front of all occurences of labels among $(\mathrm{V}|\exists| S)$ : the corresponding multiset is the set of paths $X_{G G}=(-\mid+) E^{*}(\vee|\exists| S)$.

- Kuroda double-negation translation consists the insertion of in double negation at the root and after all occurences of labels $\forall$ : the corresponding multiset is the set of paths $X_{K u}=$ $(-\mid+)\left(E^{*} \forall\right)^{?} L$.

\section{Partial orders among double-negation translations}

The main issue in the comparison of double-negations translations is the fact that the insertions of double negation do not alter the strength of judgments in the same way. Some of them weaken judgments, while other strengthen them, and the insertion of a given double negation might partially compensate the insertion of an other. A simple example of this phenomenon can be found using the judgment $\vdash(\forall x P(x)) \Rightarrow Q$. This judgment can be translated both to $\vdash(\forall x P(x)) \Rightarrow \neg \neg Q$ - this can be done using the translation presented in the following - and to $\vdash(\forall x \neg \neg P(x)) \Rightarrow \neg \neg Q$, which is stronger. The double negation on the right of the implication weakens the statement, while that on the left strengthens it. Indeed:

- $(\forall x P(x)) \Rightarrow \neg \neg Q \nvdash(\forall x P(x)) \Rightarrow Q$

- $(\forall x P(x)) \Rightarrow \neg \neg Q \vdash(\forall x P(x)) \Rightarrow \neg \neg Q$

- $(\forall x P(x)) \Rightarrow \neg \neg Q \nvdash(\forall x \neg \neg P(x)) \Rightarrow \neg \neg Q$

This shows that the second translation, which adds more double negations, allows nevertheless to compensate part of the weakening effect of the first translation.

This example shows that one crucial question when inserting a double negation at a given occurence is to predict if this double negation weakens or strengthens the original judgment. 
Fortunately, it is possible to split the set of occurences and the set of paths into the set of positive ones, at which the insertion of a double negation weakens the sequent, and the set of negative ones, at which the insertion of a double negation strengthens the sequent. This notion of polarity can be formalized in the language of paths as follows:

Definition 3. The set of directed edges $E$ can be split into the set of negative ones $E_{-}$and the set of positive ones $E_{+}$:

$E_{-}=(\Rightarrow \mathrm{L} \mid \neg)$, and $E_{+}=(\wedge \mathrm{L}|\wedge \mathrm{R}| \vee \mathrm{L}|\vee \mathrm{R}| \Rightarrow \mathrm{R}|\forall| \exists)$

Considering furthermore the antecedent position (-) as negative and the consequent position $(+)$ as positive, the polarity of an occurence is given by its path:

- The set of positive paths is $P_{+}=\left(-E_{+}^{*} E_{-} \mid+\right)\left(E_{+}^{*} E_{-}\right)^{2 *} E_{+}^{*} L$.

- The set of negative paths is $P_{-}=\left(-\mid+E_{+}^{*} E_{-}\right)\left(E_{+}^{*} E_{-}\right)^{2 *} E_{+}^{*} L$

All double negations at negative occurences, which strengthen sequents, have no use concerning the correctness of translations: if the image of a sequent by a given translation has a constructive proof, then, a fortiori, the sequent obtained from the same translation limited to positive double negations has also a constructive proof.

Therefore, it is possible to limit translations to positive occurences, leaving the choice of negative ones to the user: from a classical proof of $\Gamma \vdash(\forall x P(x)) \Rightarrow Q$, the user might choose between computing the first translation, or, if a stronger conclusion is needed, to compute first a classical proof of $\Gamma \vdash(\forall x \neg \neg P(x)) \Rightarrow Q$ from the original one before translating it, which leads to the second translation.

As a consequence, only the double negations at positive occurences should be taken into consideration when comparing the strength alteration of different translations, and furthermore, translations inserting less double negations at negative occurences can be considered better. This leads to consider that both the syntax and the strength of a sequent $S$ are less altered by a translation $f(S)$ than by a translation $g(S)$ if the set of occurences of $S$ where $f$ inserts double negations is included in the set of occurences in sequents where $g$ does, which leads to the following partial order:

Definition 4. The relation $\leq$ is defined as follow: for two double-negation translations $f$ and $g, f \leq g$ if if for any sequent $S, f$ only inserts double negations in $S$ where $g$ does. Let $f_{X}$ and $f_{X^{\prime}}$ be two syntax-directed translations. $f \leq g$ if and only if $X \subseteq X^{\prime}$.

This partial order being based on an inclusion relation, many translations are incomparable. However, at the syntax level, there is no universal way to compare double negations inserted at different positions. For instance, if a proposition $A \wedge B$ can be translated to $\neg \neg A \wedge \neg \neg B$ or to $\neg \neg(A \wedge B)$ one user could prefer the first one, which translated a conjunction to a conjunction, while another could prefer the second one, which inserts less double negations.

We suggest conversely to consider such choices as equivalent when the strength of the translation stays unchanged. Double negation commutes with connectives and quantifiers in the following cases:

Proposition 1. • $\neg \neg(A \wedge B)$ is constructively equivalent to $\neg \neg A \wedge \neg \neg B$

- $\neg \neg(A \Rightarrow B)$ is constructively equivalent to $A \Rightarrow \neg \neg B$

- $\neg \neg(\neg A)$ is constructively equivalent - and identical - to $\neg(\neg \neg A)$

The proof is based on short and straightforward constructive proofs. Proposition 1 leads to the definition of the following elementary transformations, which map correct translations to correct translations: 
- If at least one double negation is inserted at the head of a subformula $A \wedge B$, one of them is replaced by an extra double negations at the head of $A$ and an extra double negation at the head of $B$.

- If at least one double negation is inserted at the head of a subformula $A \Rightarrow B$, one of them is replaced by an extra double negation at the head of $B$.

- If at least one double negation is inserted at the head of a subformula $\neg A$, one of them is replaced by an extra double negation at the head of $A$.

We use the notation $f^{\downarrow}$ to refer to the normal form of a double-negation translation $f$ under these transformations. From this definition, an extended partial order $\leq \downarrow$ between doublenegation translations can be defined as follow:

Definition 5. The relation $\leq \downarrow$ is defined as follow: for two double-negation translations $f$ and $g, f \leq^{\downarrow} g$ if $f^{\downarrow}<g^{\downarrow}$ or if $f=g$.

It is straightforward to see that this definition corresponds also to a partial order - it is reflexive, antisymmetric and transitive. But one can also notice that if $f \leq g$ implies $f^{\downarrow} \leq g^{\downarrow}$ : therefore, $\leq \downarrow$ is an extension of $\leq$.

This extended partial order can be compared with the translations simplifications presented in [2] - which is defined in a broader context, including translations that are not strictly based on the insertion of double-negations, such as [7] - allowing to show minimal properties of some translations such as Kuroda's for instance. The extended partial order $\leq \downarrow$ leads to even lighter translations. In particular, it leads to a unique minimal syntax-directed translation, which is presented in the following section.

\section{The minimal translation}

The idea leading to the minimal syntax-directed translation is to mix the respective advantages of Kuroda's and Gödel-Gentzen's translations, limiting them to positive occurences only.

As in Kuroda's translation, we begin restricting double negations to the following set:

$$
(-\mid+)\left(E^{*} \forall\right)^{?} L
$$

Restricting it to double negations at positive occurences, this leads to:

$$
+L \mid\left(-E_{+}^{*} E_{-} \mid+\right)\left(E_{+}^{*} E_{-}\right)^{2 *} E_{+}^{*} \forall L
$$

Finally, inspired from Gödel-Gentzen's translation, we suggest to push double negations into subformulae until they reach a label among the set $G=(\vee|\exists| S)$. To be more precise, double negations can be pushed to both children for the $\wedge$ connective, and only to the right child for $\Rightarrow$; they can be pushed through $\forall$ quantifiers; they can be eliminated when they reach $\perp$, $\neg$, or $\top$ connectives. Using the set $H=(\wedge \mathrm{L}|\wedge \mathrm{R}| \Rightarrow \mathrm{R})$ of directed edges, this leads to the following set:

$$
M=+H^{*} G \mid\left(-E_{+}^{*} E_{-} \mid+\right)\left(E_{+}^{*} E_{-}\right)^{2 *} E_{+}^{*} \forall H^{*} G
$$

It will be shown in the following sections that this set defines a correct syntax-directed translation, which can be proved minimal.

One can notice that the only information used from the path to decide if a double negation must be inserted is the polarity of the occurence and, in the positive case, the presence of a root or a quantifier $\forall$ separated from the end only by directed edges of $H$. If we split the set of 
words $w \in(+\mid-) E^{*}$ according to the parity of negative elements in it, and, in the case of an even number, is in one of these three cases, according to the presence of a root or a quantifier $\forall$ separated from the end only by directed edges of $H$, the three subsets of $(+\mid-) E^{*}$ can be easily defined by mutual recursion. Following the same idea, the function $f_{M}$ can be defined by mutually recursion, using the following functions $\varphi, \chi$ and $\psi$ :
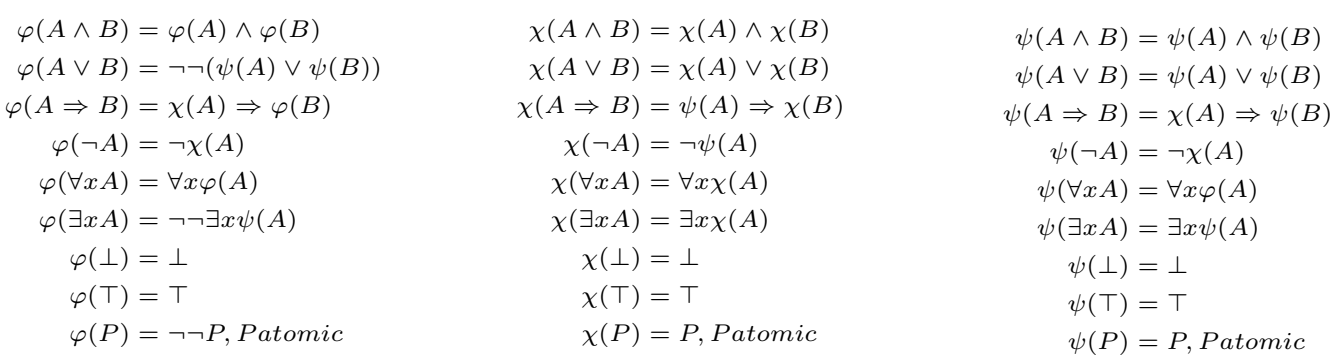

The relation between $f_{M}$ and these three functions is simply the following:

Proposition 2. $f_{M}$ is given by $\left(f_{M}^{-}, f_{M}^{+}\right)=(\chi, \varphi)$.

Proof. This proposition follows from this stronger proposition, which can be proved directly by induction: let $w \in(-\mid+) E^{*}$, let $A$ a proposition,

- $F_{X}(w, A)=\varphi(A)$ if $w \in+H^{*} \mid\left(-E_{+}^{*} E_{-} \mid+\right)\left(E_{+}^{*} E_{-}\right)^{2 *} E_{+}^{*} \forall H^{*}$, i.e. $w$ if is the longest strict prefix of some path $p \in M$

- $F_{X}(w, A)=\chi(A)$ if $w \in\left(-E_{+}^{*} E_{-} \mid+\right)\left(E_{+}^{*} E_{-}\right)^{2 *} E_{+}^{*}$, i.e. $w$ if is the longest strict prefix of some path $p \in P_{-}$

- $F_{X}(w, A)=\psi(A)$ else, i.e. $w$ if is the longest strict prefix of some path $p \in P_{+} \backslash M$.

Then, for any proposition $A, f_{M}^{-}(A)=F_{M}(-, A)=\chi(A)$ and $f_{M}^{+}(A)=F_{M}(+, A)=\varphi(A)$.

This translation is light compared to Gödel-Gentzen's and Kuroda's. We will give two examples to illustrate the differences between these translation.

First, using the classical theorem $\vdash \forall z((\exists x P(x, z)) \vee \forall x \neg P(x, z))$ :

- Gödel-Gentzen: $\vdash \forall z \neg \neg((\neg \neg \exists x \neg \neg P(x, z)) \vee \forall x \neg \neg \neg P(x, z))$

- Kuroda: $\vdash \neg \neg \forall z \neg \neg((\exists x P(x, z)) \vee \forall x \neg \neg \neg P(x, z))$

- $f_{M}: \vdash \forall z \neg \neg((\exists x P(x, z)) \vee \forall x \neg P(x, z))$

In this case, no unnecessary double negation was inserted by $f_{M}$.

Second, using the constructive theorem $\vdash \forall z \neg \neg((\exists x P(x, z)) \vee \forall x \neg P(x, z))$ :

- Gödel-Gentzen: $\vdash \forall z \neg \neg \neg \neg((\neg \neg \exists x \neg \neg P(x, z)) \vee \forall x \neg \neg \neg P(x, z))$

- Kuroda: $\vdash \neg \neg \forall z \neg \neg \neg \neg((\exists x P(x, z)) \vee \forall x \neg \neg \neg P(x, z))$

- $f_{M}: \vdash \forall z \neg \neg((\exists x P(x, z)) \vee \forall x \neg P(x, z))$ 
In this case, no double negation at all was inserted by $f_{M}$.

The latter example is also an illustration of the fact that $f_{M}$ is a projection: the set of sequents invariant by $f_{M}$ is exactly its image. The images of contexts is even lighter than the images of consequents: several finite theories as Presburger arithmetic or Robinson arithmetic are translated by themselves using this translation.

In the following section, we show that $f_{M}$ is correct.

\section{Correctness of the minimal translation}

In this section, for any function or connective $f$ mapping propositions to propositions and for any multiset of propositions $\Gamma=A_{1}, \ldots, A_{n}$, we will use the notation $f(\Gamma)=f\left(A_{1}\right), \ldots, f\left(A_{n}\right)$.

Theorem 1. $f_{M}=(\chi, \varphi)$ is a translation from classical to constructive logic: for any proposition $C$ and context $\Gamma$,

- $\Gamma \vdash C$ admits a classical proof if and only if $\chi(\Gamma) \vdash \varphi(C)$ admits a constructive one

- $\Gamma \vdash$ admits a classical proof if and only if $\chi(\Gamma) \vdash$ admits a constructive one.

Furthermore, if the original sequents admit classical proofs, the constructive proofs of the images can be computed from the original ones.

The proof is based on three lemmas.

Lemma 1. From any double-negation translation inserting double negations only at positive occurences, one can compute a proof of the image of a sequent $S$ from any proof of $S$.

The idea of the proof is the following: in the original proof, positive subformulae of the original sequent cannot appear at the root of an antecedent - they appear only inside propositions or at the root of consequents. Therefore, in order to build a proof of the image of the sequent, one can insert the rules $\neg_{\mathrm{R}}$ and $\neg_{\mathrm{L}}$ in the original proof to adapt the proof to the additional double negations as soon as they appear at the root of consequents.

Lemma 2. For any context $\Gamma$ and for any proposition $C$, if $\Gamma, \neg \varphi(C) \vdash$ admits a constructive proof, then $\Gamma \vdash \varphi(C)$ admits a constructive proof that can be computed from the original one.

Proof. This is done by structural induction on $C$. Let $\Pi$ be the proof of $\Gamma, \neg \varphi(C) \vdash$ :

- The cases of $\perp$ and $T$ are straightforward.

- If $C$ is an atom, $\varphi(C)$ is $\neg \neg C$. Then the proof can be obtained eliminating cuts in

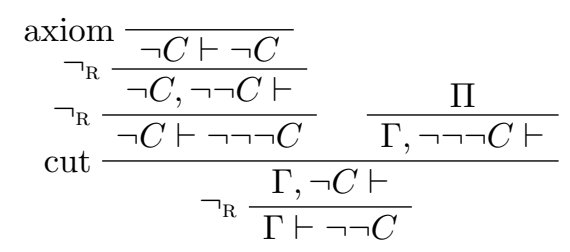

- If $C$ begins with the $\neg$ connective, the $\vee$ connective, or the $\exists$ quantifier, $\varphi(C)$ begins with a negation, which allows to use the same technique as the atomic case.

- If $C=A \Rightarrow B$, we consider the proof $\Pi^{\prime}$ obtained eliminating cuts in: 


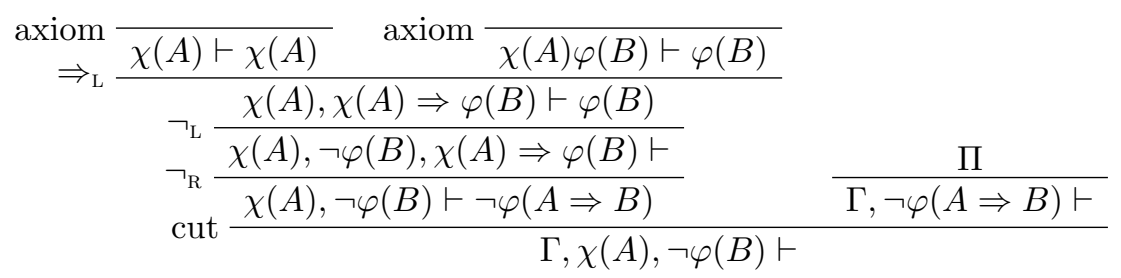

We can apply the induction hypothesis to $\Pi^{\prime}$ to get a constructive proof $\Pi^{\prime \prime}$ of $\Gamma, \chi(A) \vdash$ $\varphi(B)$. Then, we can build the proof

$$
\Rightarrow_{\mathrm{R}} \frac{\frac{\Pi^{\prime \prime}}{\Gamma, \chi(A) \vdash \varphi(B)}}{\Gamma \vdash \varphi(A \Rightarrow B)}
$$

- Else, $C$ begins with the $\wedge$ connective or the $\forall$ quantifier, and techniques similar to the case of the $\Rightarrow$ connective can be applied, introducing and eliminating cuts to get proofs for which the induction hypothesis can be applied. In the case of the $\wedge$ connective, proofs corresponding to both children will be needed.

Lemma 3. For all multisets of propositions $\Gamma$ and $\Delta$, if $\Gamma \vdash \Delta$ admits a classical proof, then $\chi(\Gamma), \neg \psi(\Delta) \vdash$ admits a constructive proof, which can be computed from the original one.

Proof. This is done by structural induction on the classical proof of $\Gamma \vdash \Delta$. In the following, we will restrict to a representative subset of cases. For readability, we only show in sequents the propositions that are active in the last rule of the classical proof - the general case follows adding $\chi\left(\Gamma^{\prime}\right), \neg \psi\left(\Delta^{\prime}\right)$ to all contexts, $\Gamma^{\prime}$ and $\Delta^{\prime}$ being the multiset of inactive propositions one the left-hand side and on the right-hand side respectively.

- Weakening, contractions, $\perp_{\mathrm{L}}$ and $T_{\mathrm{R}}$ are translated in a straightforward way.

- The axiom rule:

$$
\text { axiom } \overline{A \vdash A}
$$

Using Lemma 1, the axiom proof of $A \vdash A$ can be weakened to a proof $\Pi$ of $\chi(A) \vdash \psi(A)$, which allows to build

$$
\neg_{\mathrm{L}} \frac{\frac{\Pi}{\chi(A) \vdash \psi(A)}}{\chi(A), \neg \psi(A) \vdash}
$$

- The $\wedge_{\mathrm{L}}$ rule:

$$
\wedge_{\mathrm{L}} \frac{A, B \vdash}{A \wedge B \vdash}
$$

Getting a proof $\Pi$ of $\chi(A), \chi(B) \vdash$ from induction hypothesis, and using that $\chi(A \wedge B)=$ $\chi(A) \wedge \chi(B)$, we get the proof

$$
\wedge_{\mathrm{L}} \frac{\Pi}{\chi(A), \chi(B) \vdash}
$$

- The other left rules $-\vee_{\mathrm{L}}, \Rightarrow_{\mathrm{L}}, \exists_{\mathrm{L}}, \neg_{\mathrm{L}}$, and $\forall_{\mathrm{L}}-$, are translated exactly in the same way as $\wedge_{\mathrm{L}}$. 
- The $\wedge_{\mathrm{R}}$ rule:

$$
\wedge_{\mathrm{R}} \frac{\vdash A \quad \vdash B}{\vdash A \wedge B}
$$

Getting by induction hypothesis a proof $\Pi_{A}$ of $\neg \psi(A) \vdash$ and a proof $\Pi_{B}$ of $\neg \psi(B) \vdash$, we get the result by cut elimination of the following proof:

$$
\begin{aligned}
& \wedge_{\mathrm{R}} \frac{\overline{\psi(A), \psi(B) \vdash \psi(A)} \quad \text { axiom } \frac{}{\psi(A), \psi(B) \vdash \psi(B)}}{\neg_{\mathrm{L}} \frac{\psi(A), \psi(B) \vdash \psi(A) \wedge \psi(B)}{\neg(\psi(A) \wedge \psi(B)), \psi(A), \psi(B) \vdash}} \\
& \begin{array}{cc}
\neg_{\mathrm{L}} & \frac{\Pi}{\neg(\psi(A) \wedge \psi(B)), \psi(A), \psi(B) \vdash} \\
\neg_{\mathrm{R}} & \frac{\Pi_{B}}{\neg(\psi(A) \wedge \psi(B)), \psi(A) \vdash \neg \psi(B)} \\
\hline
\end{array}
\end{aligned}
$$

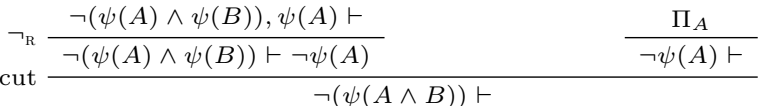

- All other right rules except $\forall_{R}$ - i.e. $\vee_{R}, \Rightarrow_{R}, \exists_{R}$, and $\neg_{R}$, are translated in the same way as $\wedge_{\mathrm{R}}$ : inserting the induction hypotheses with cuts, what remains is proved straightforwardly, and cuts can be eliminated afterwards.

- The $\forall_{\mathrm{R}}$ rule, introducing a fresh variable $x$ :

$$
\forall_{\mathrm{R}} \frac{\vdash A(x)}{\vdash \forall x A(x)}
$$

We look for a proof of $\neg \psi(\forall x A(x)) \vdash$, ie $\neg \forall x \varphi(A(x)) \vdash$. We get by induction a proof of $\neg \psi(A(x)) \vdash$ that can be weakened by Lemma 1 to a proof $\Pi$ of $\neg \varphi(A(x)) \vdash$.

From $\Pi$, we could try the technique used for the other right rules, but the proof will be stuck at the remaining sequent $\neg \forall x \varphi(A(x)) \vdash \neg \varphi(A(x))$, which is not provable - even classically. The main reason of this failure is that the technique we apply for right rules, through cut eliminations, consists in postponing the application of the rule to a deeper place in the proof. However, $\exists_{\mathrm{L}}$ and $\forall_{\mathrm{R}}$ cannot be postponed in general: they introduce fresh variables that might be necessary for the application of deeper rules $-\exists_{R}$ and $\forall_{L}$ in particular.

Therefore, as done in the case of left rules, the $\forall_{\mathrm{R}}$ has to be applied as soon as possible. This case is slightly more difficult because this $\forall_{R}$ is a right rule. It is done applying lemma 2 to $\Pi$ : this produces a proof $\Pi^{\prime}$ of $\vdash \varphi(A(x))$ which, in turn, is used to build the following proof:

$$
\forall_{\mathrm{R}} \frac{\frac{\Pi^{\prime}}{\vdash \varphi(A(x))}}{\neg \forall x \varphi(A(x))}
$$

Using these three lemmas, we prove Theorem 1 as follows:

Theorem 1. - If $\Gamma \vdash C$ admits a classical proof, then we can compute from Lemma 3 a constructive proof of $\chi(\Gamma), \neg \psi(C) \vdash$. From Lemma 1, we deduce from it a constructive proof of $\chi(\Gamma), \neg \varphi(C) \vdash$, and, from Lemma 2, we finally compute a constructive proof of $\chi(\Gamma) \vdash \varphi(C)$ 
- If $\Gamma \vdash$ admits a classical proof, then we can compute from Lemma 3 a constructive proof of $\chi(\Gamma) \vdash$ admits a constructive one.

Conversely, if such constructive proofs exist, they can be considered as classical proofs, and as any proposition is classically equivalent to its double negation, the original sequent also admits a classical proof.

The only source of complexity in $f_{M}$ comes from the use of cut eliminations. However, one can notice that the complexity is much smaller using focused proofs.

In the following section, we show the proof of minimality of $f_{M}$ according to $\leq^{\downarrow}$. The set of translations that are minimal according to $\leq-$ containing $f_{M}$ - is also identified.

\section{Minimality of the translation}

The identification of minimal translations according to the partial orders $\leq$ and $\leq \downarrow$ derives from the following theorem:

Theorem 2. Let $X$ be a multiset of paths. $f_{X}$ correct if and only if the following holds: Any path $p \in M$ can be mapped to a subpath $p_{X}$ of $p$ such that $p_{X} \in X$ and such that the directed edges completing $p_{X}$ to $p$ are all members of $H=(\wedge \mathrm{L}|\wedge \mathrm{R}| \Rightarrow \mathrm{R})$.

Before showing the details of the proof, we will focus first on its consequences:

Theorem 3. The following propositions hold:

- Let $X$ be a multiset of paths. $f_{X}$ is a minimal translation among correct syntax-directed translations according the partial order $\leq$ if and only if the following holds:

- Any path $p \in M$ can be mapped to a subpath $p_{X}$ of $p$ such that $p_{X} \in X$ and such that the directed edges completing $p_{X}$ to $p$ are all members of $H$.

$-X$ is the set union of all paths $p_{X}$.

- According to the extended partial order $\leq \downarrow, f_{M}$ is the unique minimal translation among correct syntax-directed translations.

Proof. The first proposition can be proved as follows. Let $f_{X}$ be a correct syntax-directed translation. From Theorem 2, any path $p \in M$ can be mapped to a subpath $p_{X}$ of $p$ such that $p_{X} \in X$ and such that the directed edges completing $p_{X}$ to $p$ are all members of $H$. Let $X^{\prime}$ be the set union of all paths $p_{X}$. From Theorem $2, f_{X^{\prime}}$ is also correct, and for any strict subset $X^{\prime \prime}$ of $X^{\prime}, f_{X^{\prime \prime}}$ is cannot be correct. Therefore, as $X^{\prime} \subseteq X, f_{X}$ is minimal according to $\leq$ if and only if $X^{\prime}=X$.

The second proposition can be proved as follows. As $f_{M}$ doesn't put any double negation in front of a $\Rightarrow$, a $\wedge$, or a $\neg$ connective, $f_{M}^{\downarrow}=f_{M}$. Let $f_{X}$ be a correct syntax-directed translation. From Theorem 2, any path $p \in M$ can be mapped to a subpath $p_{X}$ of $p$ such that $p_{X} \in X$ and such that the directed edges completing $p_{X}$ to $p$ are all members of $H$. Therefore, $f_{X}^{\downarrow}$ inserts double negations at any path $p \in M$, and, in case $p_{X} \neq p, f_{X}^{\downarrow}$ inserts strictly more double negations than $f_{M}$. Then, either $f_{M}<f_{X}^{\downarrow}$, either $f_{M}=f_{X}^{\downarrow}=f_{x}$. As a consequence, $f_{M}$ is minimal among correct syntax-directed translations according to the extended partial order $\leq \downarrow$.

The proof of Theorem 2 is based on the existence of sequents that ensure the existence of some paths in a multiset $X$ when $f_{X}$ is correct. In this purpose, we introduce the following notion of set of critical paths: 
Definition 6. Let $S$ be a sequent. A set $C_{S}$ of paths in $S$ is denoted as the set of critical paths of $S$ if the following holds: any double negation translation of $S$ is constructively provable if and only if it adds at least one double negation at one path of $C_{S}-$ if such a set exists, it is unique.

Using this definition, the following lemma holds.

Lemma 4. For all path $p \in M$, there exists a sequent $S$ admitting as critical paths all subpaths $p^{\prime}$ of $p$ such that the directed edges completing $p^{\prime}$ to $p$ are all members of $H$.

Proof. The proof is done step by step from particular to general cases. The first step begins with the paths in $M_{1}=+(\forall)^{?} G$ : using for instance Kripke semantics, the lemma can be proved for each one of them:

- $+P$ is the only critical path of $\neg \neg P \vdash P$,

- $+\vee$ is the only critical path of $\vdash P \vee \neg P$,

- $+\exists$ is the only critical path of $\vdash \exists x(\exists y P(y) \Rightarrow P(x))$,

- $+\forall P$ is the only critical path of $\forall z \neg \neg P(z) \vdash \forall z P(z)$,

- $+\forall \vee$ is the only critical path of $\vdash \forall z(P(z) \vee \neg P(z))$,

- $+\forall \exists$ is the only critical path of $\vdash \forall z(\exists x(\exists y P(y, z) \Rightarrow P(x, z)))$.

The second step generalizes the first one to $M_{2}=+\left(\neg^{2 *} \forall\right)^{?} G$, i.e. to add the paths $+\neg^{2+} \forall G$. Each paths $p$ of this set can be generated inserting double negations from a path $q \in+\forall G \subseteq M_{1}$. From the result of the previous step, there exists a sequent $\Gamma \vdash C$ admitting $q$ as its single critical path. Inserting in it the number $n$ of double negations that transform $q$ to $p$, the produced sequent $\Gamma \vdash \neg \neg^{2 n} C$ can be proved to admit $p$ as its single critical path.

The third step generalizes the second one to $M_{3}=+G \mid(+\mid-\neg) \neg^{2 *} \forall G$, i.e.to add the paths $-\neg \neg^{2 *} \forall G$. Each path $p$ of this set can be generated from a path $q \in+\neg^{2 *} \forall G \subseteq M_{2}$. From the result of the previous step, there exists a sequent $\Gamma \vdash C$ such that $q$ is its single critical path. This allows to prove that $\Gamma, \neg C \vdash$ admits $p$ as its single critical path.

The fourth step generalizes the third one to $M_{4}=+G \mid\left(+\mid-E_{-}\right) E_{-}^{2 *} \forall G$. Each path $p$ of $M_{4}$ can be generated from a path $q \in M_{3}$, changing some directed edges $\neg$ to $\Rightarrow \mathrm{L}$ in $q$. Let $S$ be a sequent admitting $q$ as its single path. Mapping some subformulae $\neg A$ to $A \Rightarrow \perp$ according to the transformation of $q$ to $p$, the produced sequent $S^{\prime}$ can be proved to admit $p$ as its single critical path.

The last step generalizes the fourth to $M$. Each path $p \in M$ can be generated from a path $q \in M_{4}$, adding positive directed edges to $q$. Let $S$ be a sequent admitting $q$ as its single path. Mapping some subformulae $A$ to $A \wedge \top, \top \wedge A, \top \Rightarrow A, A \vee \perp, \perp \vee A, \forall x A$, or $\exists x A$ according to the transformation of $q$ to $p$, the produced sequent $S^{\prime}$ can be proved to admit as critical paths all subpaths $p^{\prime}$ of $p$ such that the directed edges completing $p^{\prime}$ to $p$ are all members of $H$.

Theorem 2 follows from this lemma with the following proof:

Theorem 2. Let $X$ be a multiset of paths such that $f_{X}$ is correct. Let $p \in M$. From Lemma 4, there exists a sequent $S$ admitting as a critical paths all subpaths $p^{\prime}$ of $p$ such that the directed edges completing $p^{\prime}$ to $p$ are all members of $H$. As $f_{X}$ is correct, it necessarily inserts at least one double negation at one critical path: then, there exists a subpath $p_{X}$ of $p$ such that $p_{X} \in X$ and such that the directed edges completing $p_{X}$ to $p$ are all members of $H$. 
Conversely, let $X$ be a multiset such that any path $p \in M$ can be mapped to a subpath $p_{X}$ of $p$ such that $p_{X} \in X$ and such that the directed edges completing $p_{X}$ to $p$ are all members of $H$. Let $S$ be a sequent with a classical proof. From Proposition 1, the image of $S f_{X}$ is weaker of equivalent to the image $S$ by $f_{M}$ : therefore, as $f_{M}$ is correct, $f_{X}$ is also correct.

\section{Conclusion}

This paper shows that simple syntax observations are enough to get rid of many of the unnecessary double negations inserted by double-negation translations. According to a comparison of translations based on the alteration of the syntax and the strength of sequents, the problem of finding the best correct syntax-directed translation is solved, leading to the unique minimal translation $f_{M}$.

Similar techniques can be used to search the minimal translations among correct syntaxdirected translations using techniques different from double-negations, such as Friedman $A$ translation [3]. Another possible further path is to use extra tools to eliminate the double negations left by the minimal translation $f_{M}$. As mentioned earlier, constructive proof search techniques must stay avoided in the context of proof interoperability, but other ways can be followed. One of them is to analyse classical proofs in order to check, for instance, if only one subformula of a disjunction was used in its proof, or if an existential quantification was instantiated by only one term. Another possibility, when working in well-known theories, is to benefit from some of their properties. An interesting case is arithmetic: for instance, as equality is constructively decidable in this theory, decidability proofs can be used to eliminate double negations in front of all equalities. More ambitious translations from classical to constructive proofs in arithmetic could try to eliminate a larger amount of double negations, combining the presented translation with $\Pi_{2}^{0}$-conservative translations $[3,9]$.

\section{References}

[1] Gilles Dowek. On the definition of the classical connectives and quantifiers. 2013.

[2] Gilda Ferreira and Paulo Oliva. On various negative translations. arXiv preprint arXiv:1101.5442, 2011.

[3] Harvey Friedman. Classically and intuitionistically provably recursive functions. In Higher set theory, pages 21-27. Springer, 1978.

[4] Gerhard Gentzen. Über das verhältnis zwischen intuitionistischer und klassischer arithmetik. Archive for Mathematical Logic, 16(3):119-132, 1974.

[5] Kurt Gödel. Zur intuitionistischen arithmetik und zahlentheorie. Ergebnisse eines mathematischen Kolloquiums, 4(1933):34-38, 1933.

[6] Andrei Nikolaevich Kolmogorov. On the principle of excluded middle. Mat. Sb, 32(646-667):24, 1925.

[7] Jean-Louis Krivine. Opérateurs de mise en mémoire et traduction de gödel. Archive for Mathematical Logic, 30(4):241-267, 1990.

[8] Sigekatu Kuroda et al. Intuitionistische untersuchungen der formalistischen logik. Nagoya Mathematical Journal, 2:35-47, 1951.

[9] Daniel Leivant. Syntactic translations and provably recursive functions. The Journal of Symbolic Logic, 50(03):682-688, 1985.

[10] Chetan R Murthy. Extracting constructive content from classical proofs. Technical report, Cornell University, 1990. 